\title{
Residual lung lesions after completion of chemotherapy for gestational trophoblastic neoplasia: should we operate?
}

\author{
T Powles', P Savage', D Short', A Young', C Pappin' and MJ Seckl', \\ 'Department of Health Charing Cross Gestational Trophoblastic Disease Centre, Hammersmith Hospitals Campus of Imperial College London, \\ Palace Rd, London W68RF, UK
}

The significance of residual lung metastasis from malignant gestational trophoblastic neoplasm (GTN) after the completion of chemotherapy is unknown. We currently do not advocate resection of these masses. Here, we investigate the outcome of these patients. Patients with residual lung abnormalities after the completion of treatment for GTN were compared to those who had a complete radiological resolution of the disease. None of the residual masses post-treatment were surgically removed. In all, 76 patients were identified. Overall 53 (70\%) patients had no radiological abnormality on CXR or CT after completion of treatment. Eight ( I \%) patients had residual disease on CXR alone 15 patients had residual disease on CT (19\%). During follow-up, two patients (2.6\%) relapsed. One of these had had a complete radiological response post-treatment whereas the other had residual disease on CT. Patients with residual lung lesions after completing treatment for GTN do not appear to have an increased chance of relapse compared to those with no residual abnormality. We continue to recommend that these patients do not require pulmonary surgery for these lesions.

British Journal of Cancer (2006) 94, 5 I -54. doi: I 0.1038/sj.bjc.6602899 www.bjcancer.com

Published online 13 December 2005

(C) 2006 Cancer Research UK

Keywords: lung metastasis; chemotherapy; gestational trophoblastic disease

Gestational trophoblastic neoplasia (GTN) encompasses a spectrum of disorders from the premalignant conditions of complete and partial hydatidiform moles (CHM or PHM) to the malignant disorders of invasive mole, choriocarcinoma, and placental site trophoblastic tumour (PSTT). About $16 \%$ of $\mathrm{CHM}$ and $0.5 \%$ of PHM transform into any of the malignant forms of GTN. Choriocarcinoma and PSTT can also develop after any type of pregnancy, including miscarriages and term deliveries (Seckl et al, 2000; Palmieri et al, 2005). Malignant GTN is usually diagnosed by a plateaued or rising human chorionic gonadotrophin (hCG) level some weeks following uterine molar evacuation. Histological confirmation is rarely performed because of the risk of lifethreatening haemorrhage.

Malignant GTN can metastasis, most commonly to the lungs (Kumar et al, 1988). The vast majority of these patients are cured with chemotherapy despite the presence of metastatic disease, although those with liver and/or brain metastases have a poorer prognosis (Bower et al, 1997; Newlands et al, 2000). On completion of chemotherapy, most metastatic lesions will be completely resolved. However, in some patients, residual lesions persist usually in the lungs. The underlying concern is that such lesions may still harbour nests of active cancer, so should these lung lesions be removed?

\footnotetext{
*Correspondence: Professor MJ Seckl;

E-mail: m.seckl@imperial.ac.uk

Received 5 October 2005; revised 10 November 2005; accepted 14 November 2005; published online 13 December 2005
}

For nongestational choriocarcinomas, which despite being pathologically similar are genetically and clinically distinct from GTN, the answer is yes. This is because residual masses may contain active cancer regardless of normal tumour markers (Thompson and Goldstein, 1969; Gels et al, 1997; Hartmann et al, 1997a, b). However, for GTN, the situation is less clear as the disease is more chemosensitive and has an even lower relapse rate than that of nongestational choriocarcinomas arising from ovary (Sarwar et al, 2004). Therefore, our centre has historically observed residual GTN masses postchemotherapy with the exception of those in the brain, the latter being a site where chemotherapy might be less effective because of the blood-brain barrier (Newlands et al, 2002).

So is it safe to avoid resection of residual GTN lesions in the chest? Here, we attempt to address this issue by comparing the outcome for patients with or without residual radiological abnormalities in the chest, following chemotherapy for GTN.

\section{PATIENTS AND METHODS}

The Charing Cross GTN database was screened to identify all women diagnosed between 1995 and 2004, with GTN and lung metastasis as the only site of metastasis. Patients with known metastases elsewhere including the liver and brain were excluded as these sites are associated with a worse prognosis and could confound the results. Only patients whose hCG levels had normalised for 6 weeks during chemotherapy and remained normal for 6 weeks following completion of chemotherapy, and were therefore biochemically thought to be cured, were included 
in the subsequent analysis. Clinical, biochemical and radiological details (either computer tomography (CT) or chest X-ray (CXR), or both) at presentation and on completion of chemotherapy were recorded. At presentation for chemotherapy, patients were staged according to nationally and internationally accepted criteria for GTN, stratified into low- or high-risk groups, and treated accordingly (Bagshawe, 1976; Kohorn, 2001; McNeish et al, 2002). Individuals with low-risk disease were treated with subcutaneous methotrexate and folinic acid, whereas those with high-risk disease were given weekly combination chemotherapy, comprising of etoposide, methotrexate, and actinomycin alternating with cyclophospohmide and vincristine (EMA/CO). Resistance to methotrexate occurred in a minority of patients while on treatment, and therapy was changed to either actinomycin or EMA/CO depending on the level of hCG at the time of resistance as previously described (McNeish et al, 2002). During follow-up, disease-free patients outcome was recorded and plotted according to the Kaplan-Meier method. This study was approved by our local institutional review board.

\section{RESULTS}

Between 1995 and 2004, 681 patients received chemotherapy for malignant GTN. At presentation, 76 patients (11\%) had lung metastases as the only overt metastatic site, and following chemotherapy, all had normal serum hCG levels and were thought to be cured. Nevertheless, 23/76 (30\%) had persistent pulmonary radiological lesions. No pulmonary lesions were identified in the remaining 53 patients on either CXR and/or CT scanning after treatment.

The characteristics of patients with and without radiological abnormality, at the end of treatment are shown in Table 1. It is noteworthy that the median age at presentation, initial hCG, duration of treatment and proportion of patients receiving combination treatment, in the two groups are not significantly different $(P>0.05$ for each). Table 2 compares and contrasts specific factors including number and size of lung metastasis at presentation, the type of radiological investigation used at the end of treatment (CXR or CT), and the type of GTN with the risk of having persistent pulmonary lesions. Of these factors, only patients with lung lesions $>2 \mathrm{~cm}$ at the start of the treatment or those who were imaged by CT scan (rather than CXR) were found to have a significantly increased chance of residual disease on completion of treatment (Table 2). In contrast, neither the number of metastases at presentation nor the presence of confirmed choriocarcinoma altered the risk of detecting residual lesions.

Overall, two patients relapsed after chemotherapy. Both patients had nonmolar choriocarcinoma that followed term delivery. One patient had multiple metastasis at the start of the treatment, the largest of which was $5 \mathrm{~cm}$, and an hCG of $102000 \mathrm{IU} \mathrm{l}^{-1}$. She was treated with combination chemotherapy for 21 weeks. At the end of the treatment, she had three residual masses on CT scanning ranging between 1 and $1.5 \mathrm{~cm}$. Relapse occurred 3 months after the

Table I Comparability of patient groups with and without residual lung lesions following chemotherapy for malignant GTN associated with lung metastatses

\begin{tabular}{|c|c|c|c|}
\hline & All patients & $\begin{array}{l}\text { No radiological abnormality after } \\
\text { chemotherapy }\end{array}$ & $\begin{array}{l}\text { Radiologic abnormality } \\
\text { after chemotherapy }\end{array}$ \\
\hline Number & 76 & 53 & 23 \\
\hline Median age at presentation & 31 years (range: $|7-6|$ ) & 31 years (range: $|7-6|$ ) & 32 years (range: $18-58$ ) \\
\hline Median follow-up & 5 years (range: $1-9)$ & 4 years (range: $1-9$ ) & 5 years (range: $\mid-9)$ \\
\hline hCG at start of treatment & $\begin{array}{l}10900 \mathrm{IUI}^{-1}(\text { range } 150- \\
\left.2300000 \mu \mathrm{mol} \mathrm{I}^{-1}\right)\end{array}$ & $\begin{array}{l}\text { I } 10000 \mathrm{IUI}^{-1}(\text { range } 150- \\
\left.2300000 \mu \mathrm{moll}^{-1}\right)\end{array}$ & $\begin{array}{l}106000 \mathrm{IUI}^{-1}(\text { range } 170- \\
\left.\text { | } 800000 \mu \mathrm{moll}^{-1}\right)\end{array}$ \\
\hline Median duration of treatment & 18 weeks (range 10-33) & I8 weeks (rangel2-33) & 16 weeks (range 10-29) \\
\hline Proportion of high-risk patients (score $>8$ ) & $48(63 \%)$ & $33(62 \%)$ & $15(65 \%)$ \\
\hline Number of relapses & 2 & 1 & 1 \\
\hline
\end{tabular}

Table 2 Influence of CXR verses CT, size of lesion, number of lesions and type of malignant GTN on risk of having a persistent pulmonary lesion after chemotherapy

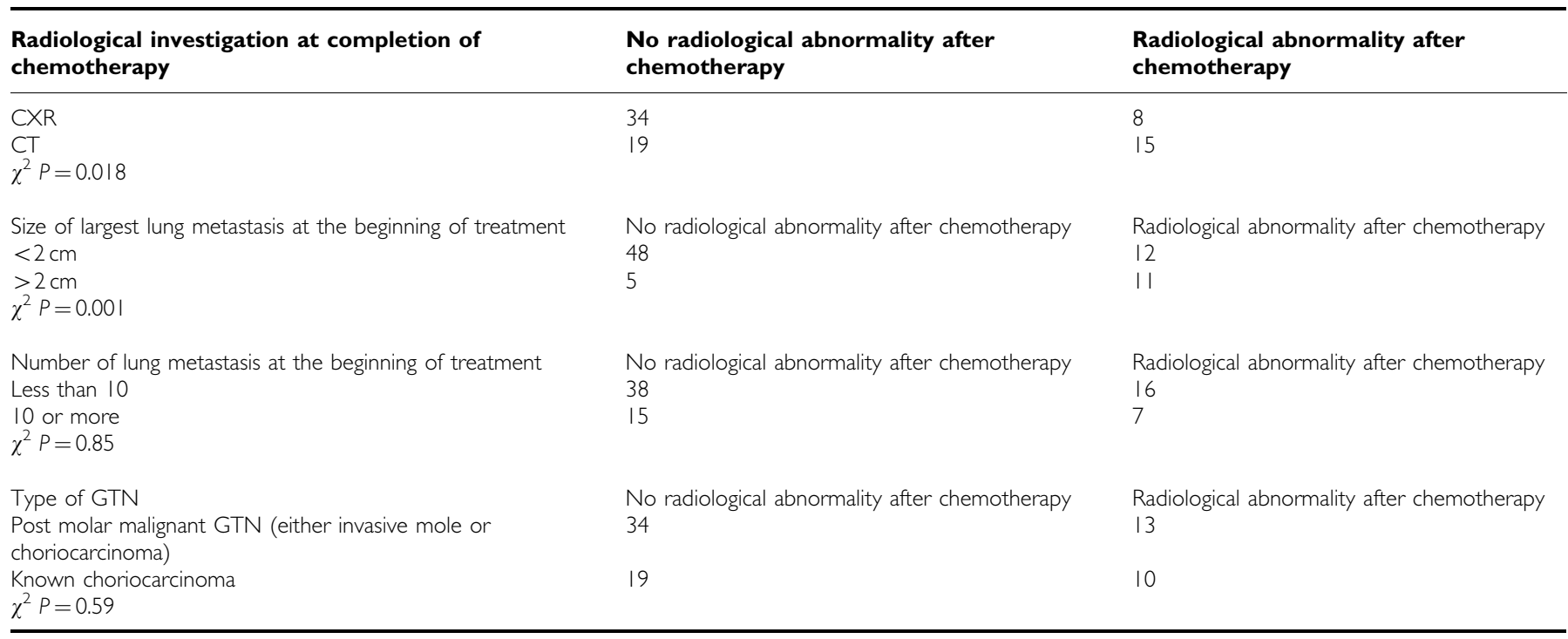




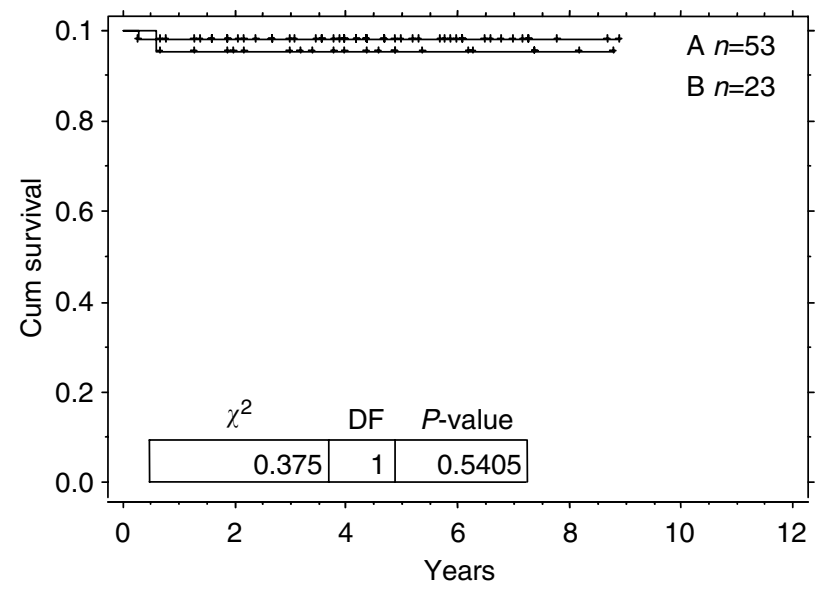

Figure I Kaplan-Meier graph for cumulative relapsed free survival. Arm $\mathrm{A}=$ no residual disease post-treatment. Arm $\mathrm{B}=$ residual disease posttreatment.

completion of treatment with a rising hCG. She went on to receive second line chemotherapy and eventually developed chemotherapy resistant disease and died from progressive GTN. The second patient had numerous $1-3 \mathrm{~cm}$ lung metastasis and an hCG of $36000 \mathrm{IU}^{-1}$ at diagnosis. She received 17 weeks of combination chemotherapy, which resulted in complete resolution of the lung metastasis on CXR. The disease relapsed in multiple sites, including the brain, 6 months later. She also eventually died from her disease.

The 5-year disease-free outcome of the whole group was $97 \%$ (95\% CI: $3-100 \%)$. For those patients with and without a residual abnormality, the 5-year disease-free survival was 95\% (95\% CI: $86-100 \%)$ and $98 \%$ (95\% CI: $94-100 \%)$, respectively.

There was no significant difference in the disease-free outcome of the patients with or without persistent radiological abnormality (Figure 1).

\section{DISCUSSION}

Little is known about the outcome of patients with residual pulmonary lesions, following successful chemotherapy for GTN with lung metastases at diagnosis. Additionally, there is uncertainty regarding the management of these residual lesions with some advocating surgical removal (Ilancheran, 1998). However, the work presented here shows that residual lung abnormalities in women who are in marker remission following chemotherapy for malignant GTN do not appear to have an increased risk of relapse. Consequently, we believe that these lesions simply represent dead tissue. Nevertheless, the absolute number of relapses in our study was small, so we cannot exclude the possibility that there may be a slight increased risk of relapse in patients with residual lung lesions when a larger cohort of patients has been studied. So is there any other data in the literature that might confirm or refute our findings?

There have been several smaller studies investigating the role of surgical resection for lung metastasis in GTN (Tomoda et al, 1980;
Saitoh et al, 1983; Xu et al, 1985; Jones et al, 1993). However, unlike our study, all these have focused on surgery in patients with an elevated hCG associated with chemoresistant tumours and many were carried out before the introduction of curative combination chemotherapy for high-risk disease. The most recent of these studies was published in 1993 (Jones et al, 1993). Seven patients had surgical resection after chemotherapy; none had a normal serum hCG at the time of surgery. Additionally, five of the patients had extra pulmonary metastasis known to be associated with a poor prognosis. Indeed, three of these patients died of extrapulmonary disease. Interestingly, two patients with isolated pulmonary metastasis were cured by surgery as others have previously reported (Sink et al, 1981). Thus, a thoracotomy in the presence of a raised hCG is only likely to be helpful for isolated chemoresistant lesions and not when there is widespread disease (Sink et al, 1981). However, none of these studies really provides any additional data to that presented here concerning the management of residual lesions when the patients are in biochemical complete remission with a normal hCG.

In our study, not all the patients had the same radiological investigation at the end of treatment. Indeed, a higher proportion of patients with radiological abnormality at the end of treatment had CT scans. This is perhaps not surprising, as CT scanning is more sensitive at identifying lung lesions than CXR. Nevertheless, our results suggest that the observation of persistent radiological abnormalities at the end of the treatment has no bearing on the outcome for these patients. Therefore, the type of radiological test performed may be irrelevant. In view of the radiation exposure related to CT scanning, we do not recommend this investigation at the end of the treatment in this young patient population. Moreover, since radiological residual abnormalities in the chest do not appear to predict subsequent disease course, and relapses can be easily serologically detected by a rising hCG, one might be tempted to advocate no chest imaging at this time. Nevertheless, we still recommend performing a follow-up CXR on completion of therapy for women with lung metastases at presentation. This is because such imaging provides a useful baseline for future comparison. For example, should the patient undergo chest imaging for other reasons years later, the finding of a lung lesion might not necessarily prompt additional investigation if comparison with the previous films postchemotherapy shows no change. In addition, should the patient relapse and a CXR demonstrate a lung lesion, it would be important to know whether this was new, enlarged from before, or old and unchanged.

Perhaps unsurprisingly, this study also shows that patients with residual disease at the end of treatment have larger lung metastasis at diagnosis (Table 2). It may be that larger pulmonary metastasis result in more nonviable tissue after the completion of treatment.

In summary, chemotherapy alone cures the majority of patients with isolated lung metastasis. Radiological abnormalities at the end of treatment are of no prognostic significance if the patient is in hCG remission, and excision of these lesions does not therefore seem reasonable.

\section{ACKNOWLEDGEMENTS}

This work was supported by the Department of Health and NTRAC.

\section{REFERENCES}

Bagshawe KD (1976) Risk and prognostic factors in trophoblastic neoplasia. Cancer 38: $1373-1385$

Bower M, Newlands ES, Holden L, Short D, Brock C, Rustin GJ, Begent RH, Bagshawe KD (1997) EMA/CO for high-risk gestational trophoblastic tumours: results from a cohort of 272 patients. I Clin Oncol 15: $2636-2643$

Gels ME, Hoekstra HJ, Sleijfer DT, Nijboer AP, Molenaar WM, Ebels T, Schraffordt Koops H (1997) Thoracotomy for postchemotherapy 
resection of pulmonary residual tumor mass in patients with nonseminomatous testicular germ cell tumors: aggressive surgical resection is justified. Chest 112: $967-973$

Hartmann JT, Candelaria M, Kuczyk MA, Schmoll HJ, Bokemeyer C (1997a) Comparison of histological results from the resection of residual masses at different sites after chemotherapy for metastatic nonseminomatous germ cell tumours. Eur J Cancer 33: 843-847

Hartmann JT, Schmoll HJ, Kuczyk MA, Candelaria M, Bokemeyer C (1997b) Postchemotherapy resections of residual masses from metastatic non-seminomatous testicular germ cell tumors. Ann Oncol 8: $531-538$

Ilancheran A (1998) Optimal treatment in gestational trophoblastic disease. Ann Acad Med Singapore 27: 698-704

Jones WB, Romain K, Erlandson RA, Burt ME, Lewis Jr JL (1993) Thoracotomy in the management of gestational choriocarcinoma. A clinicopathologic study. Cancer 72: 2175-2181

Kohorn EI (2001) The new FIGO 2000 staging and risk factor scoring system for gestational trophoblastic disease: description and critical assessment. Int J Gynecol Cancer 11: 73-77

Kumar J, Ilancheran A, Ratnam SS (1988) Pulmonary metastases in gestational trophoblastic disease: a review of 97 cases. $\mathrm{Br} \mathrm{J}$ Obstet Gynaecol 95: 70-74

McNeish IA, Strickland S, Holden L, Rustin GJS, Foskett M Seckl MJ, Newlands ES (2002) Low risk persistent gestational trophoblastic disease: outcome following initial treatment with low-dose methotrexate and folinic acid, 1992-2000. J Clin Oncol 20: $1838-1844$

Newlands ES, Holden L, Seckl MJ, McNeish I, Strickland S, Rustin GJ (2002) Management of brain metastases in patients with high-risk gestational trophoblastic tumors. J Reprod Med 47: 465-471
Newlands ES, Mulholland PJ, Holden L, Seckl MJ, Rustin GJ (2000) Etoposide and cisplatin/etoposide, methotrexate, and actinomycin D (EMA) chemotherapy for patients with high-risk gestational trophoblastic tumors refractory to EMA/cyclophosphamide and vincristine chemotherapy and patients presenting with metastatic placental site trophoblastic tumors. J Clin Oncol 18: 854-859

Palmieri C, Fisher RA, Sebire NJ, Lindsay I, Smith JR, McCluggage WG Savage P, Seckl MJ (2005) Placental site trophoblastic tumour arising from a partial hydatidiform mole. Lancet 366: 688

Saitoh K, Harada K, Nakayama H, Terauchi A, Nagano T, Inoue K (1983) Role of thoracotomy in pulmonary metastases from gestational choriocarcinoma. J Thorac Cardiovasc Surg 85: 815-820

Sarwar N, Newlands ES, Seckl MJ (2004) Gestational trophoblastic neoplasia: the management of relapsing patients and other recent advances. Curr Oncol Rep 6: 476-482

Seckl MJ, Fisher RA, Salerno GA, Rees H, Paradinas FJ, Foskett M, Newlands ES (2000) Choriocarcinoma and partial hydatidiform moles. Lancet 356: 36-39

Sink JD, Hammond CB, Young Jr WG (1981) Pulmonary resection in the management of metastases from gestational choriocarcinoma. $J$ Thorac Cardiovasc Surg 81: 830 - 834

Thompson HT, Goldstein AM (1969) Resection in the treatment of pulmonary metastases from testicular tumours and its influence on the overall survival-rate. Br J Surg 56: 349-351

Tomoda Y, Arii Y, Kaseki S, Asai Y, Gotoh S, Suzuki T, Kondoh T, Imaizumi M (1980) Surgical indications for resection in pulmonary metastasis of choriocarcinoma. Cancer 46: 2723-2730

$\mathrm{Xu}$ LT, Sun CF, Wang YE, Song HZ (1985) Resection of pulmonary metastatic choriocarcinoma in 43 drug-resistant patients. Ann Thorac Surg 39: $257-259$ 